\title{
A Swollen Heart in Pregnancy
}

\author{
Margaret C. Lo ${ }^{\mathrm{a}, \mathrm{e}}$, Jason A. Freed ${ }^{\mathrm{b}}$, \\ Li Luc, Erin M. Dunbar ${ }^{d}$
}

\begin{abstract}
Thymic carcinoma is a rare malignancy of the anterior mediastinum. Its aggressive course and early metastases portend a poor prognosis. Presentation during pregnancy increases the risk of recurrence, metastases, and maternal death. We describe a case of a healthy, pregnant female with a history of thymoma presenting with cardiopulmonary distress from recurrent pleural and pericardial effusions. The patient responded to serial thoracenteses and pericardiocenteses but extensive work-up remained nondiagnostic. CT thorax revealed an enlarging thymoma with bulky lymphadenopathies. Subsequent pathology re-analysis of the original thymoma biopsy showed pan-cytokeratin, P63, and EBV-positive epithelial tumor cells, similar to our axillary lymph node hematopathology and confirmed the thymoma as the primary malignancy. Clinicopathologic diagnosis of metastatic thymic carcinoma was finalized through multidisciplinary consensus. The patient responded well to radiation therapy but the mother and fetus died abruptly two weeks later. This case illustrates the diagnostic and therapeutic dilemmas of thymic carcinoma and its devastating consequences during pregnancy. The limited therapies in pregnancy and the sparse evidenced-based-medicine practices all contribute to poor outcomes. Attention to the differential diagnosis of anterior mediastinal tumors in pregnancy, evaluation of patients'
\end{abstract}

Manuscript accepted for publication Oct 14, 2011

${ }^{\text {a } U n i v e r s i t y ~ o f ~ F l o r i d a ~ C o l l e g e ~ o f ~ M e d i c i n e, ~ D e p a r t m e n t ~ o f ~ M e d i c i n e, ~}$ Division of Internal Medicine PO Box 100277 Gainesville, FL 32610, USA

${ }^{\mathrm{b}}$ Department of Medicine, Harvard Medical School, Beth Israel Deaconess, Boston, MA 02215, USA

${ }^{\mathrm{c}}$ Department of Pathology \& Immunology, University of Florida College of Medicine, Gainesville, FL 32610, USA

${ }^{\mathrm{d} D e p a r t m e n t ~ o f ~ N e u r o s u r g e r y, ~ U n i v e r s i t y ~ o f ~ F l o r i d a ~ C o l l e g e ~ o f ~}$ Medicine, Gainesville, FL 32610, USA

${ }^{e}$ Corresponding author: Margaret C. Lo.

Email: margaret.lo@medicine.ufl.edu

doi:10.4021/jmc375w immunocompetency and EBV-status, re-analysis of prior tissue pathology, and early multidisciplinary clinicopathologic diagnosis are all encouraged to expedite therapy and reduce mortality.

Keywords: Thymic Carcinoma; Pregnancy; Pericardial Effusion; Pleural Effusion; Syncope

\section{Introduction}

Thymomas are rare but the most common tumor of the anterior mediastinum. Less than $2 \%$ of primary thymic tumors develop into thymic carcinoma [1, 2] which accounts for only $0.2-1.5 \%$ of all malignancies [3]. Both originate from the epithelial cells of the thymus gland with no clear etiology of pathogenesis and no known risk factors. The thymus matures and releases T-lymphocytes, peaking activity during puberty then involuting. The tumor cells in a thymoma demonstrate an indolent course with a slow rate of growth, infrequent spread beyond the thymus, and similar histological appearances to normal thymus cells. On the other hand, the tumor cells in thymic carcinoma exhibit a very aggressive pattern with a rapid rate of growth, distant metastasis by the time of diagnosis and different histological appearances than the normal cells of the thymus. The common metastasis sites include the pleura, lung, liver, kidneys, bone, and axillary lymph nodes $[4,5]$. Few cases spread to the pericardium or result in pericardial effusions and cardiac tamponade. Despite its rarity and esotericism, metastatic thymic carcinoma is a clinically significant disease entity of poor prognosis especially in pregnancy with increased mortality due to its high propensity for malignancy, early metastasis, and delayed diagnosis. Here we report a devastating case of invasive thymic carcinoma in a healthy, pregnant, young female, manifesting as cardiopulmonary distress secondary to massive pericardial and pleural effusions and leading ultimately to maternal and fetal demise. We further provide a review of the literature on metastatic thymic carcinoma, its diagnostic and therapeutic challenges encountered by clinicians, and implications for diagnosis and management in clinical practice. 


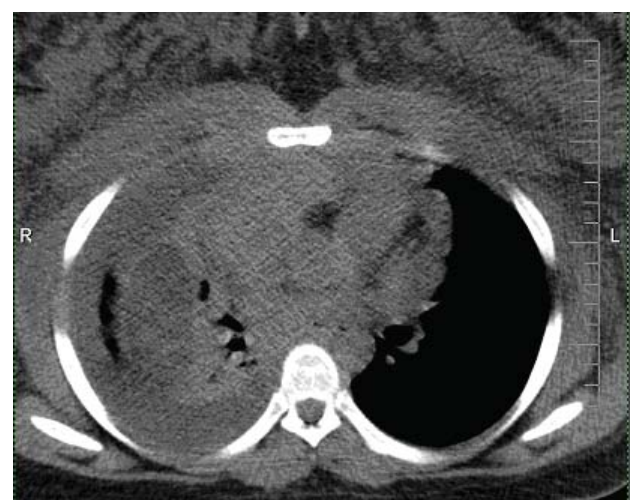

Figure 1. Non-contrasted CT thorax demonstrates large anterior mediastinal mass with associated bulky mediastinal and axillary lymphadenopathies, large right pleural effusion, and anasarca of upper chest wall and breasts.

\section{Case Presentation}

A 23-year-old, 19-week pregnant female with a history of thymoma presented as a hospital-to-hospital admission for syncope secondary to a large pericardial effusion. She denied prodromal symptoms but reported pregnancy-induced exercise intolerance and recent dyspnea on exertion. She was in excellent health with the pregnancy progressing well. She denied medications, alcohol, tobacco or illicit drug use and had no pertinent family history.

Medical history included a $10 \mathrm{~cm}$ benign thymoma per an outside biopsy 8-month ago. A CT angiogram 4 months later noted the thymoma in intimate contact with the great vessels and pulmonary arteries. Excision of the thymoma was recommended at that time, but the patient could not afford the surgery.

Physical examination revealed a healthy, comfortable African-American female who was hemodynamically stable without pulsus paradoxus or respiratory distress. Lungs were clear to auscultation with decreased breath sounds, dullness to percussion and egophony at the right bases. Cardiac examination revealed distant heart sounds and mild tachycardia but no murmurs, rubs, gallops, jugular venous distention, or pericardial knock. The head/ears/eyes/nose/throat, gastrointestinal, and neurologic examination were unremarkable with no palpable neck masses or lymphadenopathies.

Initial, outside 2 view chest X-ray (2V-CXR) showed a small right pleural effusion. EKG revealed mild sinus tachycardia and low voltage but no ST-T changes or electrical alternans. Transthoracic echocardiogram (TTE) exhibited a large pericardial effusion (14 $\mathrm{mm}$ anterior, $26 \mathrm{~mm}$ posterior) but no right heart collapse, cardiac tamponade or left ventricular dysfunction.

The next day upon hospital transfer, a repeat TTE confirmed the large pericardial effusion, now with right atrial and ventricle collapse but no tamponade. Repeat 2V-CXR showed an enlarging right pleural effusion with lung collapse. Fluid analysis revealed exudative pleural and pericardial effusions but nondiagnostic cultures, cytology, flow cytometry, and hematopathology. Work-up was negative for
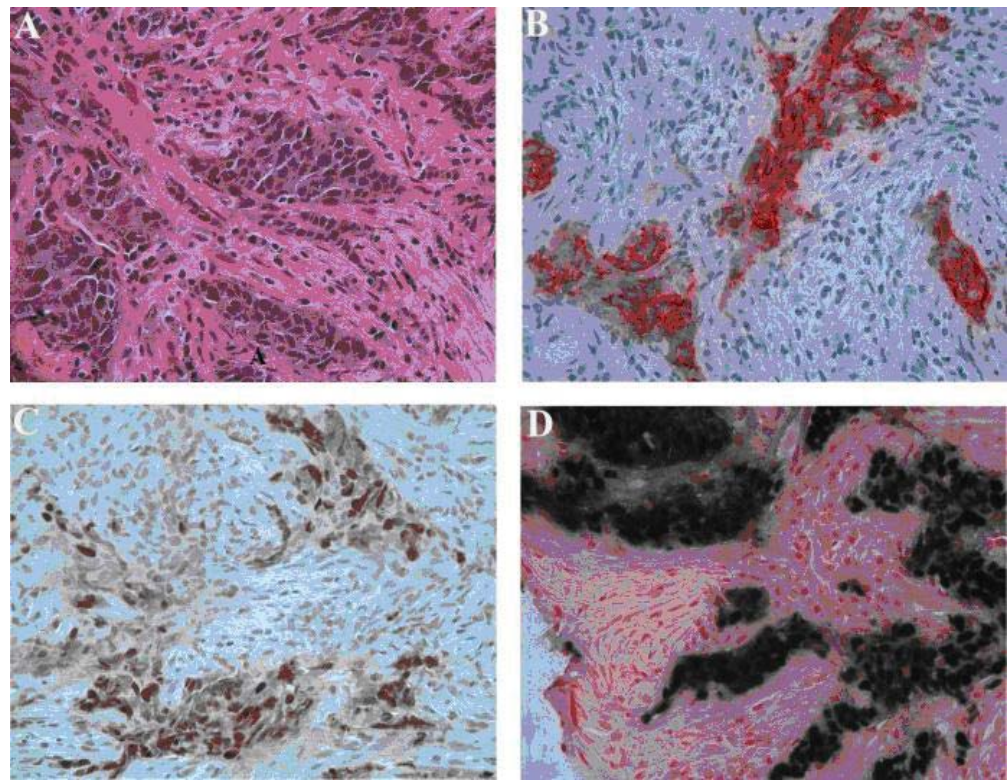

Figure 2. Left axillary lymph node biopsy. (A) Poorly differentiated carcinoma with squamous features (hematoxylin-eosin stain, original magnification, $x$ 400). (B) Tumor positive for cytokeratin (Immunostain for cytokeratin AE1/3, original magnification $\times 400$ ). (C) Positive nuclear staining for P63 (Immunostain for P63, original magnification $x$ 400) and (D) In-situ hybridization for Epstein-Barr virus shows strong positivity on tumor cells (EBER, original magnification $x 400$ ). 


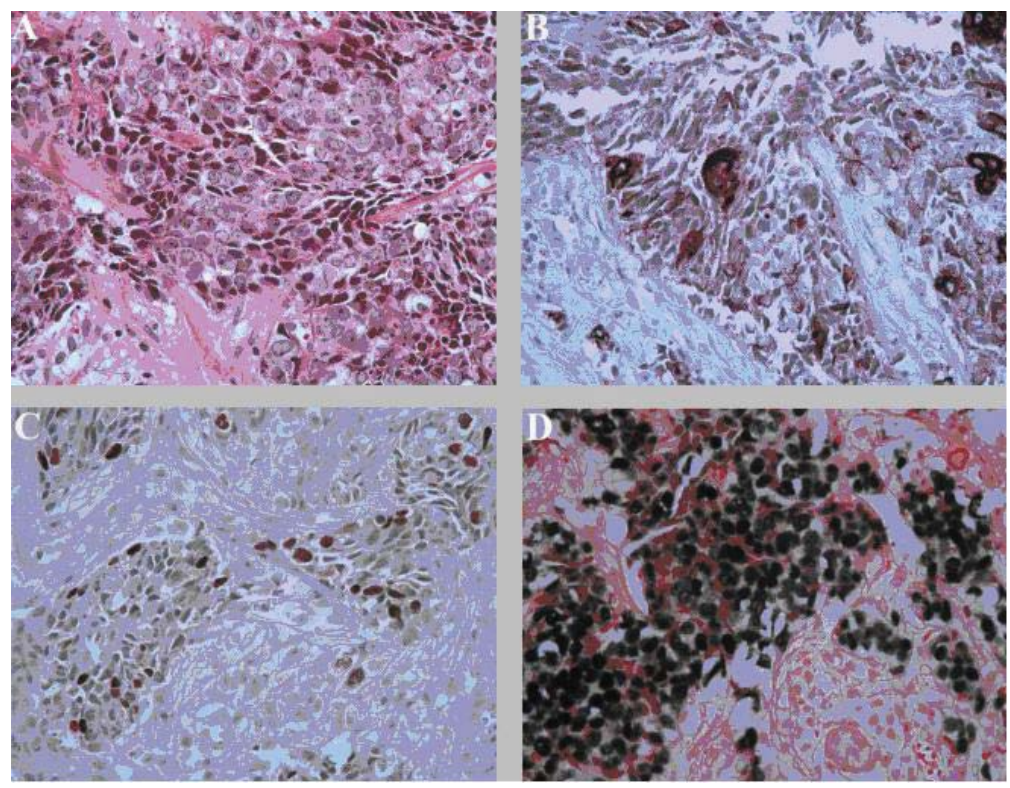

Figure 3. Anterior mediastinal mass biopsy. (A) Poorly differentiated carcinoma with squamous features (hematoxylin-eosin stain, original magnification, $x$ 400). (B) Tumor positive for cytokeratin (Immunostain for pan-cytokeratin, original magnification $x$ 400). (C) Positive nuclear staining for P63 (Immunostain for P63, original magnification $\times 400$ ) and (D) In-situ hybridization for EpsteinBarr virus shows strong positivity on tumor cells (EBER, original magnification $x 400)$.

autoimmune, endocrine, immunologic, and infectious processes including blood cultures, Epstein-Barr Virus (EBV), HIV, Cytomegalovirus, and Coxsackievirus. CT thorax and Ventilation/Perfusion scan were not performed due to the radiation risk to the fetus.

The patient responded well to serial large-volume thoracenteses and pericardiocenteses. After serial CXR and TTE demonstrated no further effusions, she requested discharge with close multidisciplinary follow-up.

Four days later, the patient returned as a hospital-tohospital readmission for moderate cardiopulmonary distress. Outside TTE showed a minimal pericardial effusion. Outside non-contrasted thorax CT revealed a large right pleural effusion, a significant increase in the thymoma size, bulky axillary and mediastinal lymphadenopathies, diffuse pulmonary nodules, and subcutaneous anasarca in the chest wall (Fig. 1). Interventional radiology (IR) performed an ultrasound-guided core biopsy of a palpable axillary lymph node. Flow cytometry showed no immunophenotypic evidence of B or T-cell lymphoproliferative disorders. Hematopathology revealed metastatic carcinoma with squamous cell differentiation, strong pan-cytokeratin and P63 immunopositivity, and EBV-positive in-situ hybridization, suggestive of nasopharyngeal carcinoma (Fig. 2). However, non-contrasted maxillofacial-head CT showed no nasopharyngeal or intracranial masses. Cardiology, Cardiothoracic Surgery, Obstetrics, IR, Pathology, and Oncology were all consulted for multidisciplinary collaboration of this diagnostic dilemma. Cardiothoracic surgery felt resection and open biopsy of the thymoma were too risky. Numerous attempts at IR-directed biopsy failed due to the patient's cardiopulmonary decompensation upon recumbency. Obstetrics felt it was too early to safely administer chemotherapy or deliver a viable baby, which was the patient's primary concern.

Later, subsequent pathology re-analysis of the initial thymoma biopsy with in-situ hybridization and immunostains showed pan-cytokeratin, P63, and EBV-positive epithelial tumor cells, similar to the carcinoma from our axillary lymph node biopsy, and confirmed the thymoma as the primary malignancy (Fig. 3). Multiple tumor board conferences at our institution and another academic center specializing in thymic carcinoma reached a multidisciplinary consensus on a clinicopathologic diagnosis of metastatic thymic carcinoma. Multidisciplinary consensus also felt thymoma surgery for further diagnostic confirmation and therapy was too risky and the best therapy was palliative radiation treatment to bulky sites with chemotherapy upon delivery of the fetus after gestational week 30. After responding well to 30-GY radiation therapy, the patient requested discharge with close multidisciplinary follow-up. Two weeks after discharge, the mother and fetus died at home from cardiac arrest.

\section{Discussion}

Thymomas, representing 20\% of anterior mediastinal tumors, and thymic carcinomas, representing $1-2 \%$ of thymomas, 
originate from epithelial cells without known pathogenesis $[3,6]$. Both can co-exist together [1] and occur in the 4th to 6th decade, either as asymptomatic with abnormal imaging or as cardiopulmonary and/or constitutional symptoms. Thymic carcinoma often presents beyond the anterior mediastinum, most commonly with pleural effusions, and EBV-positivity. Less commonly, thymic carcinoma presents with early dissemination i.e. pleural/pericardial effusions, cardiac tamponade; or during pregnancy $[1,2,4,5,7,8]$. Diagnosis usually requires FDG-PET/CT or MRI followed by tissue confirmation, a process often impeded by severe symptomology and/or pregnancy. The current dogma of clinical practice has conditioned clinicians to avoid radiographic studies in pregnant patients due to the fear of radiation risk to fetus. With the recent advances in diagnostic procedures, this reluctance to use radiographic studies in pregnancy places an undue delay on timely diagnosis and should be discouraged if maternal life is at stake.

Our case highlights the diagnostic and therapeutic dilemmas of thymic carcinoma, particularly in pregnancy and in turn, will educate clinicians on its disease manifestation and diagnostic approach for early detection and treatment. Firstly, thymic carcinoma is so exceedingly rare with nonspecific symptoms that many clinicians lack the clinical experience to recognize its disease presentation. Diagnosis involves consideration of an extensive differential diagnosis of anterior mediastinal masses, including lymphomas, parathyroid tumors, intra-thoracic thyroid tissue, germ cell tumors, and congenital abnormalities [6]. The differential diagnosis of pleural/pericardial effusions, anasarca, and diffuse lymphadenopathy in pregnancy is equally wide and technically challenging. Diagnostic pursuits must focus on the patient's immunocompetency and the absence of infectious or rheumatologic risk factors. This directed us away from HIV-associated malignancies and infectious or rheumatologic etiologies and toward malignancies of young adults worsened by pregnancy, such as thymic carcinoma. Secondly, the pathophysiology of thymic carcinoma is poorly understood. It is associated with EBV-positivity, a well-known accelerator and discriminator of malignancy in many epithelial cancers [9]. In this case, confirmation of EBV-positivity in both the lymph node and original thymic tissue was essential. Interestingly, literature often report both EBV-positivity and pregnancy to accelerate thymic carcinoma [10-12] and estrogen receptor staining to correlate with increased tumor size, clinical stage, and the World Health Organization histologic classification [13]. These reports shed some light to possible targets for pathophysiology research. Thirdly, diagnosis in pregnancy is challenging given numerous physiologic changes including anasarca and hormonal fluctuations. Pregnancy further limits the location and size of tissue sampling for diagnosis, two well-known negative prognostic factors [1, $2,14]$. The patient's pregnancy, severe symptomology, and limited tissue availability were significant roadblocks to diagnosis in this case. For us, the patient's symptomatology and the correlation between the current lymph node biopsy and the original thymic tissue biopsy aided our pathology and oncology colleagues in finalizing the diagnosis of metastatic thymic carcinoma. It is essential for clinicians to act promptly on an early multidisciplinary clinicopathologic diagnosis in pregnancy to minimize unnecessary tests and expedite therapy $[4,5,7]$.

Thymic carcinoma follows an aggressive course with a poor prognosis despite intensive therapy $[15,17]$. No ideal therapy currently exists for widely-disseminated thymic carcinoma. Initial therapy commonly includes surgery +/- radiation and salvage chemotherapy $+/$ - radiation, although disease rarity precludes defined therapy guidelines. Given the absence of prospective randomized trials, clinicians rely on retrospective case reports for clinical management with primary focus on aggressive surgical resection for non-metastatic disease and radiation or chemotherapy for metastases and recurrence [3].

Presentation during pregnancy portends an increasingly dismal prognosis. Several cases in pregnancy report increased risk of recurrence, propensity of metastases and maternal demise [10-12, 18, 19]. The rapid disease progression in pregnancy, the limited therapies in pregnancy, and the sparse evidenced-based-medicine practices all contribute to poor outcomes in pregnancy. Given such grim scenarios, older literature supports prophylactic abortions to preserve maternal life [19, 20]. In our case, termination of pregnancy was indeed discussed with the patient who adamantly declined this option. Clinicians can best advocate for their pregnant patients by recognizing the devastating consequences of metastatic thymic carcinoma during pregnancy and promptly counseling their pregnant patients toward an informed decision on appropriate diagnosis and therapy.

\section{Learning points}

Thymic carcinoma carries a poor prognosis due to its lack of evidenced-based therapies and high propensity for malignancy, early metastasis, and delayed diagnosis. This case serves to educate clinicians on the diagnostic and therapeutic dilemmas encountered in disseminated thymic carcinoma and further highlights its aggressive course and overwhelming challenges during pregnancy. Clinicians must pay close attention to the differential diagnosis of anterior mediastinal tumors in pregnancy and the evaluation of patients' immunocompetency and EBV-status. Reanalysis of prior tissue biopsy, if applicable, is essential to confirm the accuracy of the current pathology at hand. Early clinicopathologic diagnosis by multidisciplinary collaboration is also strongly encouraged. This case adds to the collective wisdom in the literature on optimal diagnostic and therapeutic approaches of thymic 
carcinoma, including clinical consideration on whether aggressive therapy will benefit pregnancy outcomes.

\section{Acknowledgments}

We would like to thank all the Blue Medicine team members involved in caring for this patient and contributing to the manuscript.

\section{Conflicts of Interest}

The authors have no relevant academic and financial disclosures or conflicts of interests and have listed their outside activities separately. All authors meet authorship criteria and participated fully in the write-up and submission of the manuscript. The authors attest that this manuscript is an original work with no prior publication of manuscript contents and is not under consideration for publication elsewhere.

\section{Funding source}

None.

\section{References}

1. Suster S, Rosai J. Thymic carcinoma. A clinicopathologic study of 60 cases. Cancer. 1991;67(4):1025-1032.

2. Sperling B, Marschall J, Kennedy R, Pahwa P, Chibbar R. Thymoma: a review of the clinical and pathological findings in 65 cases. Can J Surg. 2003;46(1):37-42.

3. Fornasiero A, Daniele O, Ghiotto C, Sartori F, Rea F, Piazza M, Fiore-Donati L, et al. Chemotherapy of invasive thymoma. J Clin Oncol. 1990;8(8):1419-1423.

4. Muller-Hermelink HK, Marx A. Pathological aspects of malignant and benign thymic disorders. Ann Med. 1999;31 Suppl 2:5-14.

5. Liu HC, Hsu WH, Chen YJ, Chan YJ, Wu YC, Huang BS, Huang MH. Primary thymic carcinoma. Ann Thorac Surg. 2002;73(4):1076-1081.

6. Neal MP, Jr. Physiologic approach to diagnosis of mediastinal disease. South Med J. 1971;64(5):517-527.
7. Lewis JE, Wick MR, Scheithauer BW, Bernatz PE, Taylor WF. Thymoma. A clinicopathologic review. Cancer. 1987;60(11):2727-2743.

8. Eng TY, Fuller CD, Jagirdar J, Bains Y, Thomas CR, Jr. Thymic carcinoma: state of the art review. Int J Radiat Oncol Biol Phys. 2004;59(3):654-664.

9. Leyvraz S, Henle W, Chahinian AP, Perlmann C, Klein G, Gordon RE, Rosenblum M, et al. Association of Epstein-Barr virus with thymic carcinoma. N Engl J Med. 1985;312(20):1296-1299.

10. Massart JJ, Bishop WA. Thymoma occurring during pregnancy. A case report. Obstet Gynecol. 1968;32(4):490-493.

11. Goldman KP. Malignant thymoma in pregnancy. Br J Dis Chest. 1974;68:279-283.

12. Huang CC, Lee CC. Thymoma and myasthenia gravis in pregnancy: report of a case. J Formos Med Assoc. 1991;90(2):206-208.

13. Ishibashi H, Suzuki T, Suzuki S, Moriya T, Kaneko C, Takizawa T, Sunamori M, et al. Sex steroid hormone receptors in human thymoma. J Clin Endocrinol Metab. 2003;88(5):2309-2317.

14. Wilkins KB, Sheikh E, Green R, Patel M, George S, Takano M, Diener-West M, et al. Clinical and pathologic predictors of survival in patients with thymoma. Ann Surg. 1999;230(4):562-572; discussion 572-564.

15. Davenport E, Malthaner RA. The role of surgery in the management of thymoma: a systematic review. Ann Thorac Surg. 2008;86(2):673-684.

16. Singhal S, Shrager JB, Rosenthal DI, LiVolsi VA, Kaiser LR. Comparison of stages I-II thymoma treated by complete resection with or without adjuvant radiation. Ann Thorac Surg. 2003;76(5):1635-1641; discussion 16411632.

17. Maruyama R, Suemitsu R, Okamoto T, Kojo M, Aoki Y, Wataya H, Eguchi T, et al. Persistent and aggressive treatment for thymic carcinoma. Results of a single-institute experience with 25 patients. Oncology. 2006;70(5):325-329.

18. Argubright KF, Mattox JH, Messer RH. Thymoma in pregnancy. Obstet Gynecol Surv. 1984;39(4):185-191.

19. Peleg D, Zabari A, Shalev E. Relapsing thymic carcinoma during pregnancy. Acta Obstet Gynecol Scand. 1992;71(5):398-400.

20. Drews EF, Ungeheuer E. Termination of pregnancy from the surgical viewpoint. Med Klin. 1971;66(1):1-8. 